\title{
Seeing a tree through the forest: Precision medicine tools can enhance donor allocation in heart transplantation
}

\author{
Vishnu Vasanthan, MD, and Paul W. M. Fedak, MD, PhD, FRCS(C)
}

\author{
From the Section of Cardiac Surgery, Department of Cardiac Science, Cumming School of Medicine, University \\ of Calgary, Libin Cardiovascular Institute of Alberta, Calgary, Alberta, Canada. \\ Disclosures: Authors have nothing to disclose with regard to commercial support. \\ Received for publication Nov 18, 2017; accepted for publication Dec 1, 2017; available ahead of print Jan 5, 2018. \\ Address for reprints: Paul W.M. Fedak, MD, PhD, FRCS(C), C880, 1403 29th St NW, Calgary T2N 2T9, Alberta, \\ Canada (E-mail: paul.fedak@gmail.com). \\ J Thorac Cardiovasc Surg 2018;155:1591-2 \\ $0022-5223 / \$ 36.00$ \\ Copyright (C) 2017 by The American Association for Thoracic Surgery \\ https://doi.org/10.1016/j.jtcvs.2017.12.008
}

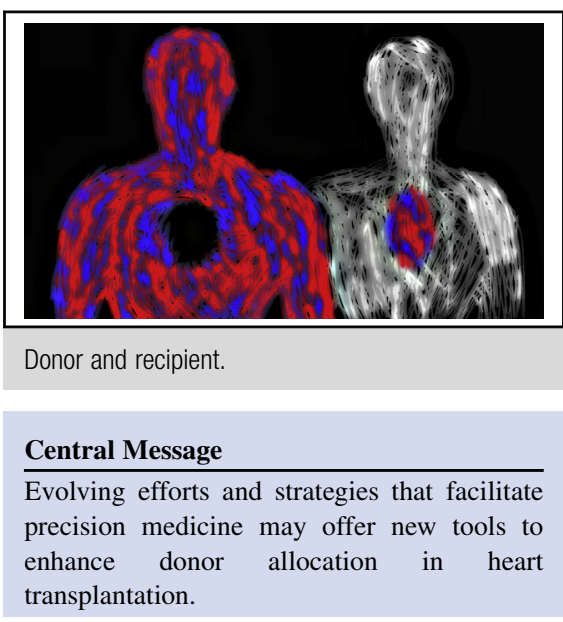

See Article page 1580.
Health care paradigms are shifting toward precision medicine approaches to personalize management on the basis of an individual patient's physiologic, biochemical, and genetic profiles. ${ }^{1}$ Efforts at "big data" are expanding largescale clinical databases ${ }^{2,3}$ to facilitate development of specific criteria that can identify individuals at high risk within populations. Personalized treatment strategies are vital to success within a resource-limited system. Even as we are counseled to see a "forest through the trees," to optimize the use of a limited therapy we are challenged to do the opposite and find a specific tree (optimal donor organ) within the forest (pool of candidates).

Mortality and outcomes in cardiac surgery are improving, yet heart transplantation remains limited by a paucity of suitable donor organs and subsequent waiting list mortality. ${ }^{4}$ With a limited donor pool and clinical momentum to act, clinicians are challenged to make personalized decisions with limited information to optimize postoperative outcomes by appropriate donor organ allocation. Given the endless points of contact between donor and recipient risk factors, this is a difficult challenge.

In this issue of the Journal, Joyce and colleagues ${ }^{5}$ leverage the United Network for Organ Sharing database to identify both recipient and donor parameters that reflect statistically significant relationships with 1-year posttransplant survival. These factors are incorporated into an elegant risk assessment tool that stratifies patients into low-, medium-, and high-risk groups to facilitate pairing high-risk recipients with higher quality donors to improve outcomes. This score identifies additional modifiable risk factors that clinicians can address with the potential to mitigate risk and improve outcomes.

The proposed tool has potential to improve donor selection. Clinical judgment will always be necessary for donor allocation decisions, but the proposed tool may serve as a useful addition in difficult cases by providing a framework for discussion. In support, Joyce and colleagues ${ }^{5}$ show that at least $15 \%$ of transplants would have been deemed excessively high risk. This suggests that the quantifiable risk assessment tool had the potential to influence the discussion and perhaps the outcomes in selected cases. Overall, Joyce and colleagues ${ }^{5}$ provide the heart transplant community with a framework that can aid in clinical decisions and be further optimized in future iterations.

The development of a formal risk assessment score that reflects donor and recipient interactions may indeed improve precision. With a constantly growing database such as that of the United Network for Organ Sharing, algorithms could be created to identify critical interactive effects as predictors of mortality. Through supervised machine learning, ${ }^{6}$ the amassed data could create a continuously updated survival assessment tool that accesses the United Network for Organ Sharing database and suggests donor-recipient pairing options to help physicians optimize organ allocation. In addition, ex vivo heart perfusion is an alternative donor organ preservation modality that can be used not only to perfuse and recondition donor hearts but also to evaluate them in real time. ${ }^{4}$ In doing so, more comprehensive data could be gathered to study biochemical and physiologic cardiac parameters that affect mortality and possibly organ allocation decisions. The future use of such precision medicine tools with big data applications will most certainly be relevant to enhance heart transplant donor allocation decisions.

\section{References}

1. National Research Council (US) Committee on a Framework for Developing a New Taxonomy of Disease. Toward Precision Medicine: Building a Knowledge 
Network for Biomedical Research and a New Taxonomy of Disease. Washington (DC): National Academies Press; 2011.

2. Srinivas TR, Taber DJ, Su Z, Zhang J, Mour G, Northrup D, et al. Big data, predictive analytics, and quality improvement in kidney transplantation: a proof of concept. Am J Transplant. 2017; 17:671-81.

3. Raghupathi W, Raghupathi V. Big data analytics in healthcare: promise and potential. Health Inf Sci Syst. 2014;2:3.
4. White CW, Ambrose E, Müller A, Li Y, Le H, Hiebert B, et al. Assessment of donor heart viability during ex vivo heart perfusion. Can J Physiol Pharmacol. 2015;93:893-901.

5. Joyce DL, Li Z, Edwards LB, Kobashigawa JA, Daly RC. Predicting 1-year cardiac transplant survival using a donor-recipient risk assessment tool. J Thorac Cardiovasc Surg. 2018;155:1580-90.

6. Sun D. Development of new diagnostic techniques-machine learning. Adv Exp Med Biol. 2017;1010:203-15. 